\title{
Research on Algorithm Schema of Parametric Architecture Design Based on Schema Theory
}

\author{
Li Zhu', a , Nan Zhang ${ }^{2, b}$ and Xiaoying Qing ${ }^{3, c}$ \\ ${ }^{1}$ Room 410, No.405 Xiao Xiang Road, Yue Lu District, Changsha, China \\ ${ }^{2}$ Room 410, No.405 Xiao Xiang Road, Yue Lu District, Changsha, China \\ ${ }^{3}$ Room 108, No.71 Jiu Du West Road, Jian Xi District, Luoyang, China \\ a2413858174@qq.com, b307579716@qq.com, c270827463@qq.com
}

Keywords: Schema, Parametric Architecture, Algorithm Schema, Sensory-Motor Schema, Architecture Mechanism

\begin{abstract}
This paper mainly demonstrates the characteristics of the algorithm schema which constructs the generative mechanism of parametric architecture. By elaborating the schema theory in architecture design and the algorithm of parametric architecture design, it explicates that the algorithm has the same abstract logic structure as the schema. This structure projects the logic of architecture generation onto algorithm schema through our cognition about schematic structure, which can help us develop architectural form. In this paper, the method of using algorithm schema to develop the generated designs of architectural form has also been explored.
\end{abstract}

\section{Introduction}

As our cognition about architectural generative mechanism changes, architecture design keeps changing. In this objective world, some factors which are related to architecture generation will affect the cognition about architectural mechanism, such as context, geographical conditions, construction technologies and other things. Schema is an abstract structure extracted from our cognitive experience of the world. It could provide us with a correct cognition about the relationship of objective things. Based on schema theory, we could describe the generation of architectural mechanism as a process that architects project the abstract logic relationship relating to the factors that affect architecture generation through their own cognitive experience onto the logic of architectural form, and thus form the architectural form.

As an avant-garde design method, parametric design promotes the re-development of architectural design. Benefited from development of computer science, this design approach use a computer to set reasonable parameters (value of lighting, flow of people, visual line, sight, etc.) and then simulates various influencing factors in architecture generation circumstance as well as the relationship between them with abstract logic of schema and thus form architectural form. Buildings generated in this way generally have continuous changeable flow pattern, which implies complex parameter logic. In the nonlinear scientific perspective, this form is more adaptive to the complex environment than the Modernist functional and spatial division. Professor Xu Weiguo, Tsinghua University, claimed, "In fact, parametric architecture design is quantitative design, that is to say, design is controlled by the parameters, each parameter controls or shows some important properties. The change of parametric value leads to the change of design.”

The mechanism of parametric architecture is established by the algorithm schema. Algorithm is a concept in computer science. It has a strong correlation with schema and architectural mechanism because they all have the same abstract logic. 
Schema is an abstract structure and it is an important way to generate a concept. Human have inborn cognitive structure. The world famous psychologist of Switzerland Piaget put forward the idea that as children grow up, they have accepted more and more complex information and concepts. These concepts will continuously stimulate their brains so that they will gradually generate a dynamic cognitive structure. This is so-called schema. Our cognition about the world is from the establishment of these schemas. During the early period of the human cognition development, sensory-motor schema, which forms the basis of our cognition structure, generates us the initial cognition structure about space structure and spatial direction.

Based on the schema theory, we could project the logic structure among factors affecting the generation of architecture onto the architectural mechanism. The concept of architecture archetype in architectural typology, in a sense, is a schema. According to Peter Eisenman, the theory of transformational generative grammar is based on the logical deduction of the geometrical shape, it is also a schema. The architectural pattern, which is the most important concept of the architectural pattern language theory, is also a cognitive structure and it is a schema. In a word, schema is closely associated with architectural mechanism. As shown in Fig.1

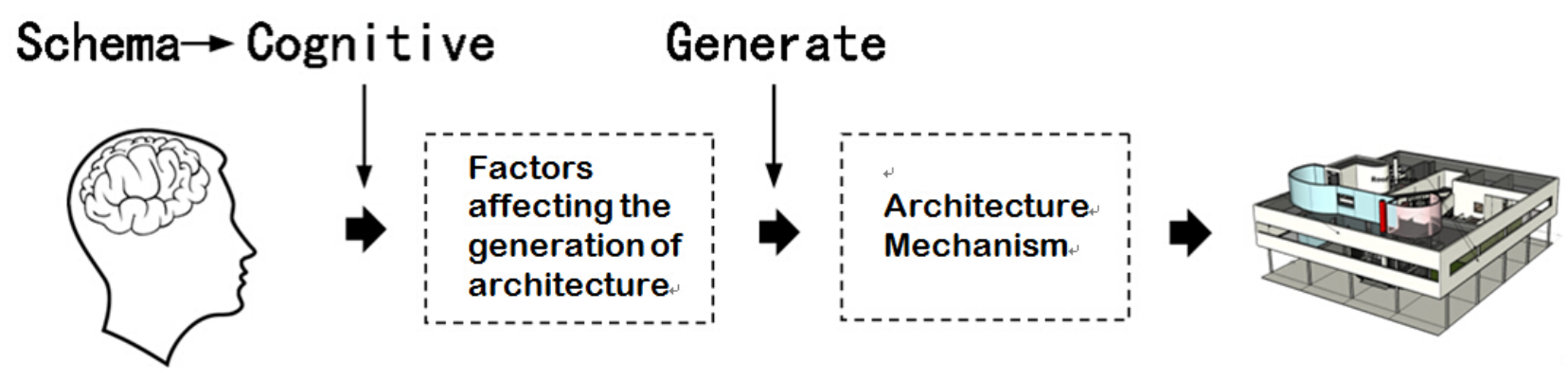

Fig. 1 Construction of Architecture Mechanism Based on Schema Theory

\section{2. “Algorithm Schema” -- a cognitive schema to construct generative mechanism of parametric architecture}

With the introduction of schema theory, we firstly conduct a schematic cognition about the structure of factors affecting the architecture generation and then transform this abstract cognitive structure into the logic of computer algorithm. In the generation process of parametric architecture, the establishment of cognitive structure is a subjective schema cognition that affects architectural formation elements objectively, algorithm schema is a projection of our cognitive schema structure by algorithm logic in the abstract computer language. In this way, we established the parametric generative mechanism of architecture form, as shown in Fig.2.

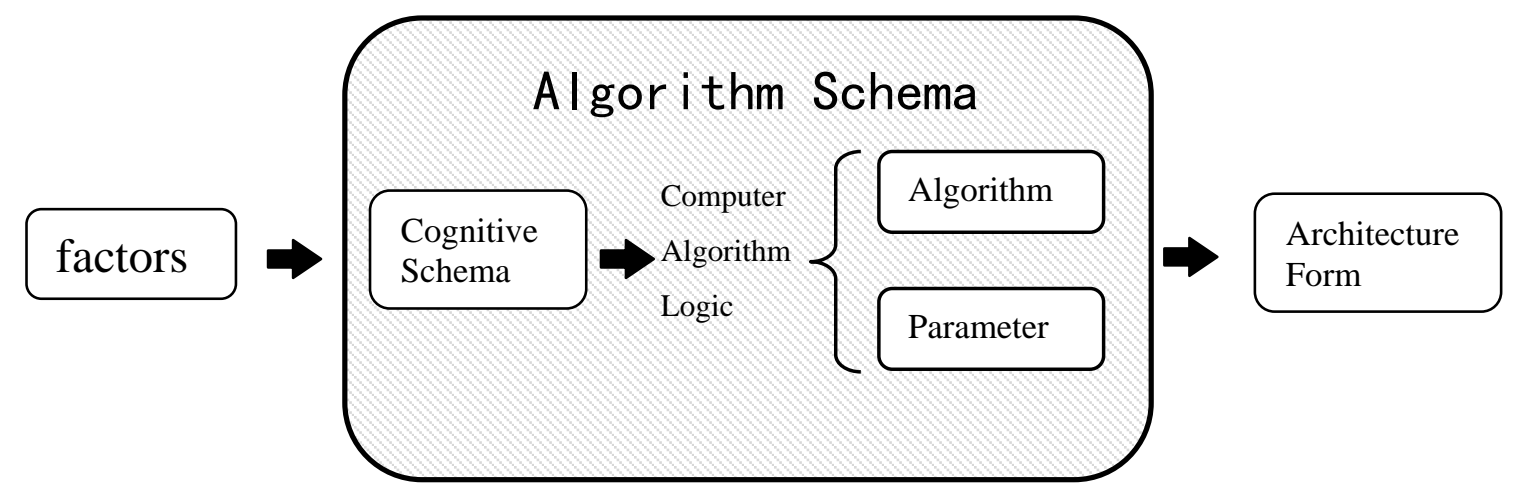

Fig. 2 Using Algorithm Schema to Establish the Parametric Generative Mechanism of Architecture 
Algorithm is a series of instructions with which we solve problems. It extracts logical principals and strategies systematically during the procedure and thus establishes perfect solutions. Algorithm itself has many properties, such as deduction, induction, abstraction, generalization and strong organizational logic. It is just like when we cook a meal, we need to buy food, prepare and then cook it, this set of procedures construct the schema of cooking food.

The schema of parametric architecture is to describe and reflect the environment which affects architecture generation with computer language. It is an approach to control the generation of architecture as well. Algorithm schema is based on parameter or logic to generate architecture, during the process of architecture generation by computer algorithm; we can classify the factors which affect the generation of architecture form through its own structure traits, and make it correspond to the objects described by data and Boolean.

As architecture design is presented as a result of its spatial form, we should transform our cognition structure about architectural generation into computer schema, that is to say, quantify abstract schema into data, parameter and Boolean. The key point is how to turn abstract cognition into quantitative space form? Here we need to introduce the foundation of the cognition structure: Sensory-Motor schema. It includes some basic schemas such as container schema, path schema, force schema, attraction schema and so on. We formed our complicated cognition about the world by these basic schemas, thus we account that the schema has a property of quantifying the abstract cognitive logic.

\subsection{Container Schema}

Just like when we describe the status between couples, we may say: "they are in love", the word "in" implies that we use the typical container schema of sensory-motor schema to perceive abstract love. Actually in our daily life, we are captured in containers: home is a cozy and warm container, vehicles are fashionable and effective containers, and office room is social communication container. This space structure is deeply internalized in our cognition structure and thus generates the schema, meanwhile project the schema structure onto new conceptions. It will help us perceive and comprehend them. Love is a abstract notion. We project the container schema onto conception of love. It could arouse our happy and enjoyable feelings just like we are in "home container", and then help us understand the abstract conception of love. We can also project the structure of container schema onto abstract structure of things and their relationship. For example, human being's behavior may produce influence on environment, we can consider people as a dot in an abstract way, and the range of its influence generates a container schema. When they move, they could influence other people and objects inside the container. After we extracting schema during this process, we can project it onto algorithm schema, to compile and construct algorithm schema. Utilizing algorithm of "metaballs" in the grasshopper, quantifying the influence produced by human beings as radius, the more influence it has, the longer radius it will be. When the distance between people is less than a constraint value, it can be considered that communicative action appeared between this two people. The influence range of this two will integrate into one single object, as is shown in Fig.3. 

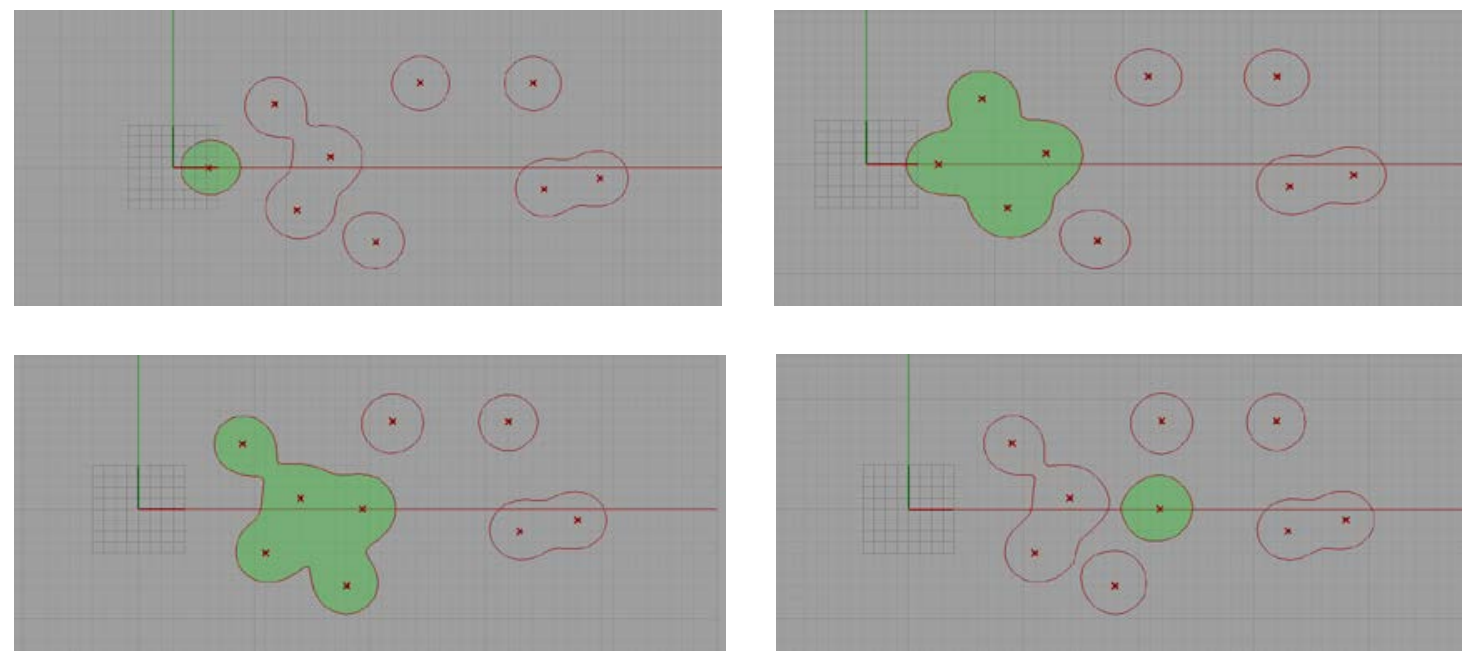

Fig. 3 The Algorithm Schema of the Influence Produced by Human Behavior

\subsection{Force Schema}

Everything in the world is subject to the effect of gravity so as not to leave away from the ground. Consequently, in our cognitive structure, force is also a kind of basic sensory-motor schema which displays a certain relationship between the giver of force and the stressed part, thus the schema of forces provides us a kind of cognitive structure so that we can understand some other concepts, such as: behavior phenomenon of human beings, physical phenomenon and cultural phenomenon.

For example, when we plan people's flow of a park landscape, the relationship between the landscape node and visitors can be projected in the way of a force schema. People attracted by the landscape node will move towards it, which can be regarded as visitors being given traction by the node. During the process of visitors' movement, the distance between them and the node changes constantly, and due to people's self-awareness and preferences, different landscape nodes would also have different traction on people with disparate aesthetic demands. In this way, a more real people flow can be formed. As shown in Fig.4.
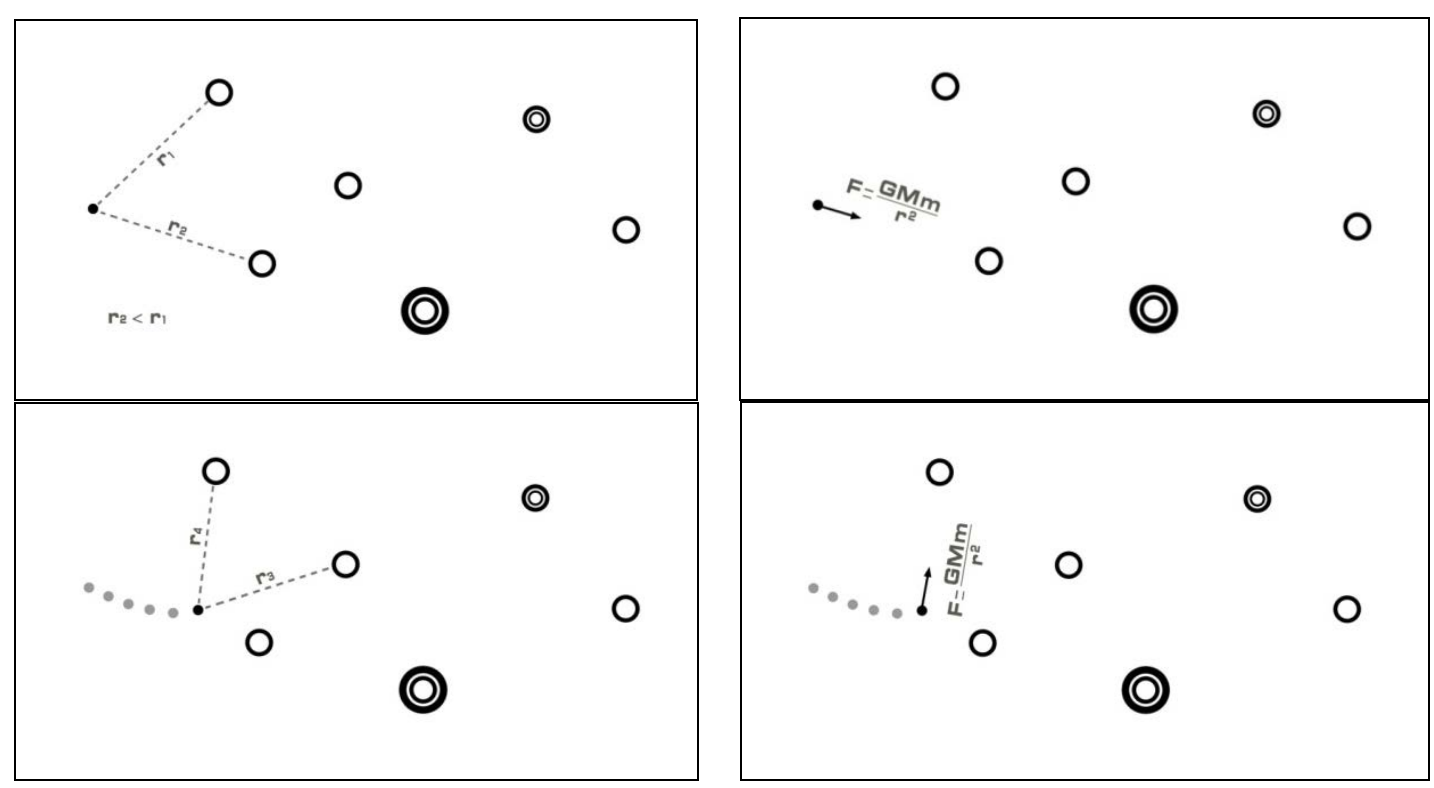

Fig.4 Force Schema in the Planning of People Flow

We can use scripting language to describe this schema structure in Rhino. Pick a dot representing a person, and set a landscape node position in the field, there is a certain force between them, the value of which can be described through gravity formula. Under the action of the traction, there occurs a displacement, and the speed of the displacement is determined by the distance between the person 
and landscape node. In this algorithm, we can simulate the walking paths of different people in the field. With multiple iterative operations, a random people flow can be obtained, and then a prototype of people flow planning can be established, as is shown in Fig.5.
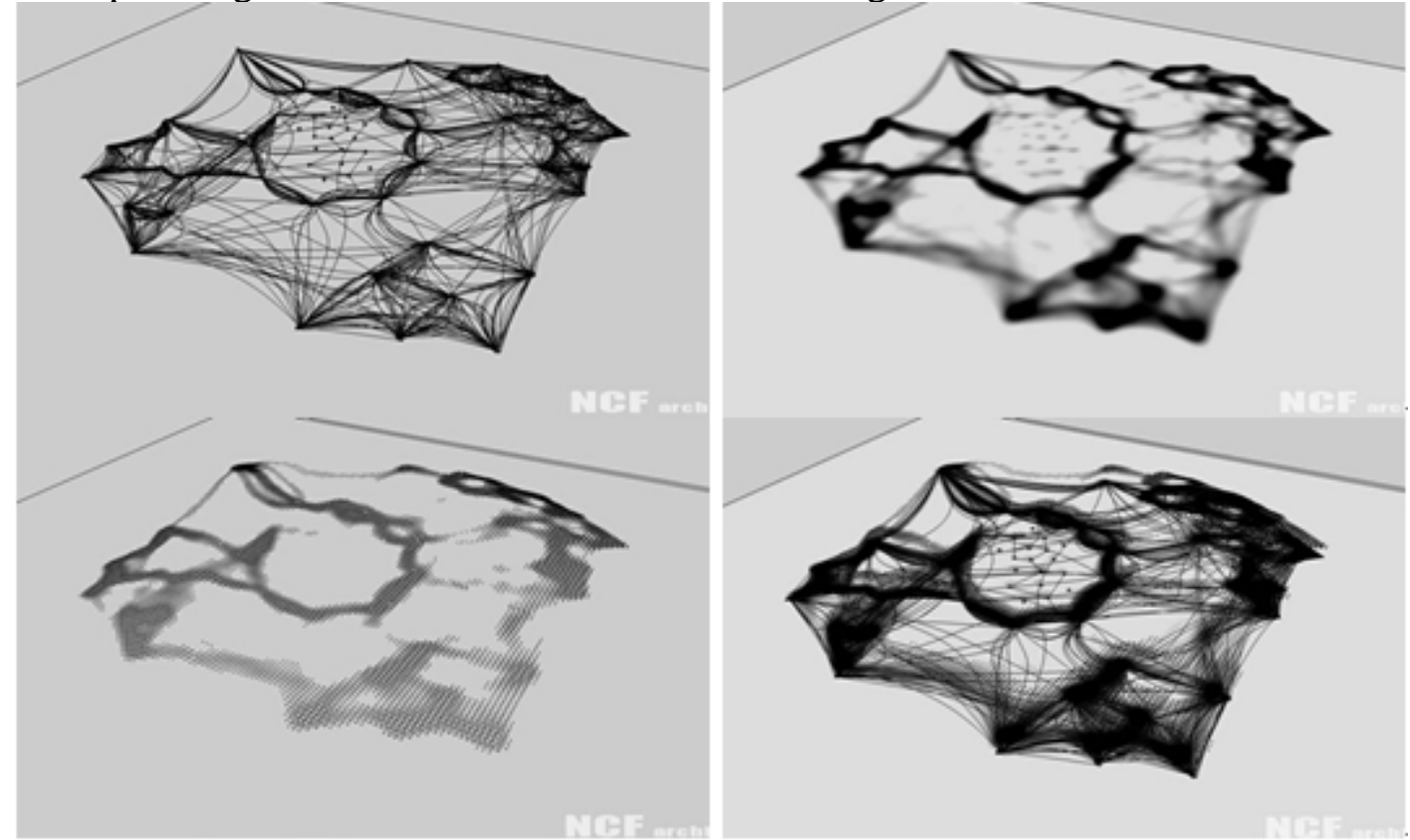

Fig.5 People Flow Trajectory Model after Multiple Iterative Operation

\subsection{Combination of Complex Algorithm Scheme}

In the process of compiling computer algorithm schema, in addition to the above two basic applications of sensory-motor schema, we can also use others to get a cognition of generating mechanism of buildings, and establish an algorithm schema. The factors that have influences on architectural form are complex and changeable, and the factors themselves are multiple-layered. Consequently, when we project this complex abstract structure onto the algorithm schema, the algorithm scheme will also present a complex nest and combination

Nested schema has been mentioned in the simple case described above. As for the planning of people flow, in addition to the use of schema structure of forces to give an explanation about the relationship between people flow and landscape node, we also adopted another basic sensory-motor schema --path schema. The combination of the two becomes the algorithm schema of people flow. The combination with the characteristics of nested schema enables us to have a profound cognition and construction of more complex generation mechanism of buildings.

\section{Summary}

In this paper, an important method of generating parametric architecture mechanism has been discussed, that is algorithm schema. We use the relevance of algorithm schema to generate logic through the cognitive schematic structure of architecture generation mechanism and parametric architecture form. With the help of the basic schema of cognitive structure - - sensory-motor schema, we quantify abstract logical relationship of architecture generation elements. In this way, spatial form based on human's cognition of architectural generative mechanism becomes operable and the parametric design no longer just stays at a superficial level, but into the construction of the architecture spatial form.

\section{Figures}

Fig. 1, self-drawn by author 
Fig. 2, self-drawn by author

Fig. 3, self-drawn by author

Fig. 4, self-drawn by author

Fig. 5, www.ncf-china.com

\section{References}

[1] J. Piaget: The Principles of Genetic Epistemology,(Rout ledge and K. Paul, London 1972)

[2] Yaoyong Qin: Image Schema-the Construction and Construal of Metaphor, Journal of Yulin Normal University, Vol. 26 (2005), p. 74-77

[3] Weiguo Xu: Architectural Creation and Thinking of the Parametric Design in China: Interview with Professor Xu Weiguo \& Xu Feng of School of Architecture of Tsinghua University, Urban Environment Design, Vol. 35 (2009), p. 108-113

[4] Tianyu Zhang, Yukun Zhang, Di Wang: Space Organization of Architecture and the Psychological Schemata, Huazhong Architecture, Vol. 24 (2006), p. 65-67

[5] Information on http://www.ncf-china.com/forum.php 